\title{
Wireless Sensor Network for m-Healthcare Monitoring of Human Being
}

\author{
Radhika Rani Chintala ${ }^{1}$, Ch N S M Akhilesh ${ }^{2}$, N P P Ganesh ${ }^{3}$, T Ravideep ${ }^{4}$ \\ ${ }^{1}$ Koneru Lakshmaiah Education Foundation, India, radhikarani_cse@kluniversity.in \\ ${ }^{2}$ Koneru Lakshmaiah Education Foundation, India, ch.n.s.m.akhilesh@ gmail.com \\ ${ }^{3}$ Koneru Lakshmaiah Education Foundation, India, ganeshnandamuri27@ gmail.com \\ ${ }^{4}$ Koneru Lakshmaiah Education Foundation, India, ravideepcse1972@gmail.com
}

\begin{abstract}
In this paper, we are going to develop an m-health care monitoring for a human being using a wireless sensor network. By using certain Wearable sensors, we detect abnormal and unforeseen situations by monitoring physiological parameters along with other symptoms. Our fundamental target is to actualize a checking framework which screens the Heartbeat, Temperature, and Humidity of the patient. This work presents a simple to-utilize framework for the quick checking of the Heartbeat. The gadgets are utilized as models. The simple sign is given to the Analog-to-Digital Converter (ADC) of Arduino. There, simple voltages are being changed over to advanced, and that computerized qualities will be put away in the EEPROM of Arduino. The qualities put away in EEPROM will be sent to the Wi-Fi module, which refreshes the cloud server like clockwork. From that server, anybody can get to that information through things speak site and see in android/ios application
\end{abstract}

Key words: Humidity, Body Temperature, Heartbeat, Arduino, EEPROM, Things speak

\section{INTRODUCTION}

We are in an era where wearable sensors are playing a massive role in our day to day life. Almost we are using them in every field, such as medical, entertainment, security, and commercial fields [1]. And as a good cause, the role of these in the medical sector is quite good and appreciating thing which we also used for the same reason where these sensors are helpful to save human lives with giving messages which alert us about the patients' health condition measuring Heartbeat, temperature, and so on. We can do this by managing the physical activities of a person who has this wearable sensor. The case of IoT devices incorporates advanced machinery, RFID labels, actuators, sensors, and cell phones. Most of the smart devices are low-resource constraint devices described with low processing power, restricted battery supply, less area, additionally little memory quantity. In these devices, information handling and conventions are deliberately intended to reach stringent activity essentials. Indeed, even with arrange application layer

Security upgrades, these dangers, and difficulties are progressively essential, especially when the low-resource constraint devices swap over sensitive information [2].

\section{FRAMEWORK AND THE PRINCIPLE OF M- HEALTHCARE MONITORING SYSTEM}

The critical designing of the human movement checking method can be explained with the help of a block diagram (figure 1).

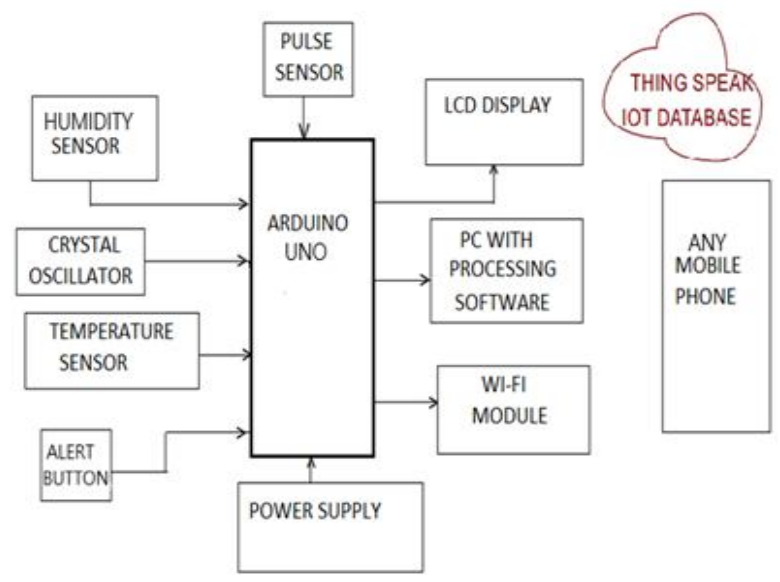

Figure 1: Block Diagram of the m-Healthcare Monitoring System

Considering the job of checking, various kinds of sensors are utilized [3]. Sensors are used to collect unrefined data from processors. The data by then picked up from the processors can show. Almost any type of essential wearable gadgets that are used by people during their exercises and while playing sports various appliances where the customers can see the recordings of the sensor if the machine has the component of remote data 
broadcast capacity, the information can be transferred to a central station all the way through a handset, the square graph representation of elementary wearable remote gadgets has shown up [4]. The data may potentially be simple at the identifying end, yet by far, most of the data is taken care of, dealt with in the PC, and accurate introduction of information is possible in two particular ways graphical or a substitute technique for the numerical worth. Considering the different kinds of natures, the yield can be obtained from the remote spot through the site [5]. The square diagram representation of a made anatomical checking structure shows up in figure 1 . The checking scheme may include a range of sensors in the direction of measuring physiological constraint, for instance, body temperature level, heartbeat; the image of the wearable physiological watching structure has shown up. The system measures skin temperature by using a temperature sensor, heartbeat sensor likewise accelerometers to recognize any drop so as to take place [6]. The entire deliberate anatomical statistics is collected by a microcontroller to analyze and process, taking care of data the central regulator might moreover show an advice message to the guardian figure predicated on the back and forth movement physiological condition of the individual seen just as could benefit in the direction to recognize early treatment.

\section{SENSORS USED FORM-HEALTHCARE MONITORING}

At present, a review of two or three sensors that are generally used for watching different person's actions undergoes. The sensor is a basic component of the entire checking framework and intended to measure physiological parameters accurately along with dependably over long length [7]. The quick improvements of microelectronics, coordinated optics, micromechanics, and further related advances have empowered the advancement of mixed sorts of distinctly intellective sensors to detect and compute information all the extra proficiently plus increasingly speedy, by lower vitality utilization as well as less handling assets. The internal heat level is one among everyday physiological parameters evaluated via wearable sensors intended for human movement conditions just as for different things, for example, action grouping. Estimating the Heartbeat can be valuable in discovering the patient's wellbeing conditions by finding in the varieties of the heartbeat levels of a patient [8]. Estimating Heartbeat is something essential so as to discover the diseases identified with a heart like a stroke. Accordingly, we can take preventive measures to fix persistent sickness.

\section{SENSOR NETWORKS FORM-HEALTHCARE MONITORING}

The design of sensors in addition to the establishment of the sensors frameworks of HAM structure accept an essential activity for exact checking of body temperature, heartbeat rate and doing in these we should consider some factors such as cost of sensors, the accuracy of sensors, and the range of sensors that should detect body parameters [8]. There may be various exceptionally designated frameworks on which research is starting at now encountering a framework designing has existed. The improvement of fall recognizable proof structures depends on the mixing of the sensor's framework, and robots have been accessible [9]. The sensor composes configuration that incorporates a worn body sensor and including sensors scattered in nature as shown in figure 2.

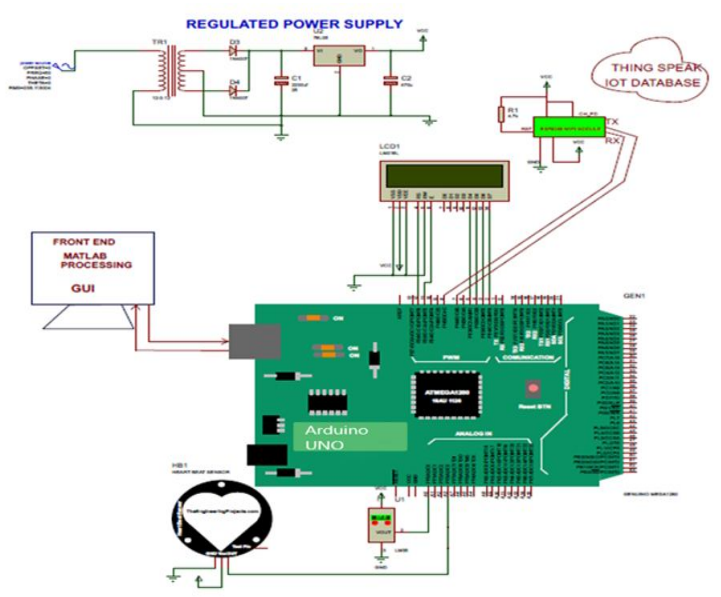

Figure 2: Circuit Diagram of m-Healthcare Monitoring

\section{VARIOUS APPROACHES OF THE SENSOR SYSTEMS}

There can be an astonishing number of exercises to be watched utilizing wearable sensors in addition to indicated ones; the approaches will besides be exceptional [10]. The information as of challenging to know, lightweight in addition to force-fit wearable sensors can utilize to see progression plans while implementing different exercises, the typical frameworks for improvement depend upon managed getting the hang of requiring primary extents of checked arranging information [11]. It is a great test to acquire precisely and point by point comments of exercises that prevent the applicability of these methodologies in certifiable settings. Another learning planbased technique for movement acknowledgments that generously decreases the necessary measure of explanation and adequately influences such inadequately named information together with more effectively possible unlabeled information has been accounted for [12]. Here we used two different sensors named DHT11, which can be used to find humidity and temperature. And the pulse sensor is used to find the Heartbeat. With the help of the DHT11 sensor, we can find only the degrees above 0 in order to find the minus degree temperature we need to use sensors DHT21 and DHT22 [13].

\section{ISSUES AND CHALLENGES IN DESIGN}

The examination plus developed investigators are attempting in the direction of structure plus make insightful wearable contraptions to be there utilized in favor of constant checking of 
a range of human exercises for 24 hours and 7 days out of consistently [14]. An epic no direct improvement structure has been familiar with considering security and requirements so as to rely on human being physiology as well as choose structure stage course of activity limitation in favor of wearable sensors application. Within remote wearable sensors, various data sources make-instance advancing traffic [15]. The size of which may be gigantic accomplishing one-sided laziness. This is a brute test to ensure that the most basic data can, all around, be passed on in a reliable way. Also, data transmission may encounter the tricky impacts of significant obfuscating and packages accidents as a result of the dynamic on-body channel incited by upgrades [16].

\section{IMPLEMENTATION AND RESULTS}

As figure 3 and figure 4 shows the DHT11 sensor is used to find the patient's humidity and temperature. It can also be used to find the outside temperature of an environment when the sensor is not connected to the patient [17]. The pulse sensor is used to find the Heartbeat of a patient as shown in the figure 5. With the help of a buzzer that is connected to a microcontroller, we can get the emergency alerts of a patient when the heartbeat rate is not normal or usually above 90 BPM. With the help of ESP 8266, which is a Wi-Fi module, we can post the data collected from a patient in the thingspeak cloud from which anyone can view the data in a public channel. The whole code for the UNO board is coded in the Arduino software tool in our system [18]. Which will then be embedded into the Arduino UNO then the working of every single sensor is done.

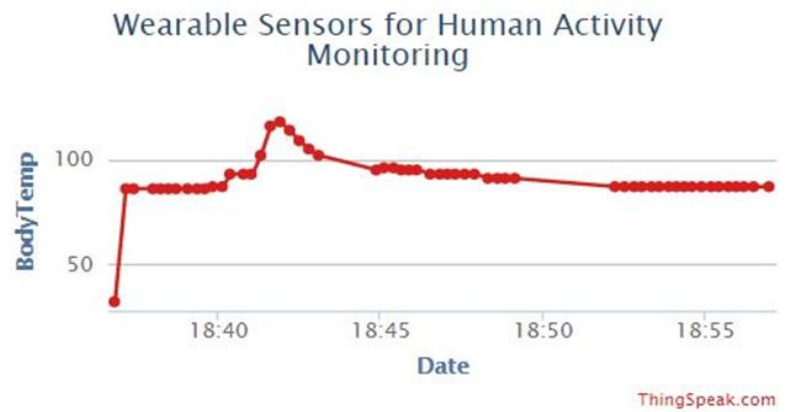

Figure 3: Graph of the Body Temperature reading Wearable Sensors for Human Activity Monitoring

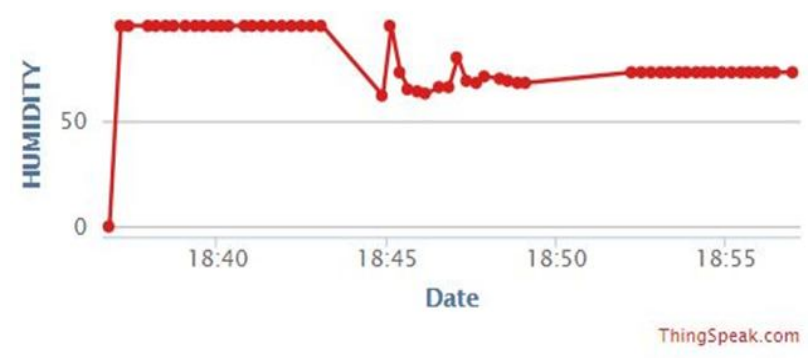

Figure 4: Graph of Humidity reading
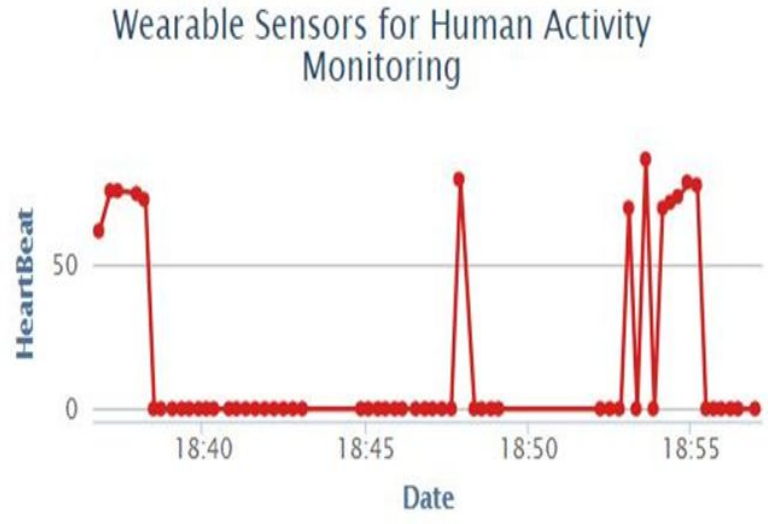

ThingSpeak.com

Figure 5: Graph of the Heart rate of human being

\section{CONCLUSION}

Human progress watching is a vivacious domain of research, and a vast amount of business progression is represented [19]. It is typical that a ton continuously lightweight, first-class the declared composition on wearable sensors and contraptions for checking drove procedure will be accessible for watching a broad extent of actions. The test looked through the existing structure determination in like manner be tended to in upcoming devices, the progression of lightweight physiological sensors might provoke social wearable widgets to screen unlike extents of actions of tenants [20]. The informal and formal survey expects the development of attention as well as following employments of wearable contraptions as soon as possible. The charge of the devices is furthermore predictable to drop coming to fruition in full function in the overall population.

\section{REFERENCES}

1. J. Edwards, "Wireless sensors relay medical insight to patients and caregivers [special reports]," IEEE Signal Process. Mag., vol. 29, no. 3,pp. 8-12, May 2012. https://doi.org/10.1109/MSP.2012.2183489

2. K. Malhi, S. C. Mukhopadhyay, J. Schnepper, M. Haefke, andH.Ewald, "A Zigbee-based wearable physiological parameters monitoring system," IEEE Sensors J., vol. 12, no. 3, pp. 423-430, Mar. 2012. https://doi.org/10.1109/JSEN.2010.2091719

3. P.A.Shaltis, A. T. Reisner, and H. H. Asada, "Cuffless blood pressure monitoring using hydrostatic pressure changes," IEEE Trans. Biomed.Eng., vol. 55, no. 6, pp. 1775-1777, Jun. 2008. https://doi.org/10.1109/TBME.2008.919142

4. B. Mariani, M. C. Jiménez, F. J. G. Vingerhoets, and K. Aminian, "On-shoe wearable sensors for gait and turning assessment of patients with Parkinson's 
disease," IEEE Trans. Biomed. Eng., vol. 60, no. 1,pp. 155-158, Jan. 2013.

https://doi.org/10.1109/TBME.2012.2227317

5. B.-R. Chen et al., "A web-based system for home monitoring of patients with Parkinson's disease using wearable sensors," IEEE Trans.Biomed. Eng., vol. 58, no. 3, pp. 831-836, Mar. 2011.

https://doi.org/10.1109/TBME.2010.2090044

6. P.Castillejo, J. F. Martínez, J. Rodríguez-Molina, and A. Cuerva,"Integration of wearable devices in a wireless sensor network for an E-health application," IEEE Wireless Common., vol. 20, no. 4,pp. 38-49, Aug. 2013.

7. Mothe, Saiteja. (2020). "A Model for Assessing the Nature of Car Crashes using Convolutional Neural Networks". International Journal of Emerging Trends in Engineering Research. 8. 859-863. doi: 10.30534/ijeter/2020/41832020.

8. C. Ranhotigmage, "Human activities and posture recognition:Innovative algorithm for highly accurate detection rate," Dept. Eng. Electron. Computer. Syst. Eng., M.S. thesis, Massey Univ., Palmerston, New Zealand, 2013.

9. T. Shany, S. J. Redmond, M. R. Narayanan, and N. H. Lovell, "Sensors-based wearable systems for monitoring of human movement and falls," IEEE Sensors J., vol. 12, no. 3, pp. 658-670, Mar. 2012. https://doi.org/10.1109/JSEN.2011.2146246

10. Prasad, M.V.D. \& Inthiyaz, Syed \& Maddala, Teja\&Kunapy, Hari \& Manohar, Minna\&Kumari, Rupa\&Shaik, Hasane. (2019). "Human activity recognition using Deep Learning". International Journal of Emerging Trends in Engineering Research. 7. 536-541. doi: 10.30534/ijeter/2019/227112019.

11. M.-Z. Poh, N. C. Swenson, and R. W. Picard, "Motiontolerant magnetic earring sensor and wireless earpiece for wearable photo-plethysmography," IEEE Trans. Inf. Technol. Biomed., vol. 14, no. 3,pp. 786-794, May 2010.

12. T. T. Zhang et al., "Sound based heart rate monitoring for wearable systems," in Proc. Int. Conf. Body Sensor New., Jun. 2010,pp. 139-143.
13. A. Inomata and $Y$. Yaginuma, "Hassle-free sensing technologies for monitoring daily health changes," FUJITSU Sci. Technol., vol. 50,no. 1, pp. 78-83, Jan. 2014.

14. Y.-C. Kan and C.-K. Chen, "A wearable inertial sensor node for body motion analysis," IEEE Sensors J., vol. 12, no. 3, pp. 651-657,Mar. 2012. https://doi.org/10.1109/JSEN.2011.2148708

15. L. T. D'Angelo, J.Neuhaeuser, Y. Zhao, and T. C. Lueth, "SIMPLE-use-Sensor set for wearable movement and interaction research," IEEE Sensors J., vol. 14, no. 4, pp. 1207-1215, Apr. 2014.

16. Y. Chuo et al., "Mechanically flexible wireless multisensor platform for human physical activity and vitals monitoring," IEEE Trans. Biomed.Circuits Syst., vol. 4, no. 5, pp. 281-294, Oct. 2010. https://doi.org/10.1109/TBCAS.2010.2052616

17. "M-Healthcare monitoring Using the Wearable Sensors in Health care" International Journal of Science, Engineering and Technology Research (IJSETR), Volume 5, Issue 2, February 2016.

18. L. Yan, J. Bae, S. Lee, T. Roh, K. Song, and H.-J. Yoo, "A $3.9 \mathrm{~mW} 25$-electrode reconfigured sensor for wearable cardiac monitoring system," IEEE J. Solid-State Circuits, vol. 46, no. 1, pp. 353-364,Jan. 2011

19. J. Cheng, O.Amft, G. Bahle, and P. Lukowicz, "Designing sensitive wearable capacitive sensors for activity recognition," IEEE Sensors J.,vol. 13, no. 10, pp. 39353947, Oct. 2013.

https://doi.org/10.1109/JSEN.2013.2259693

20. E. Y. Song and K. B. Lee, "IEEE 1451.5 standard-based wireless sensor networks," Advances in Wireless Sensors and Sensor Networks(Lecture Notes in Electrical Engineering), vol. 64. Berlin, Germany:Springer-Verlag, 2010, pp. 